\title{
A Putative Effector CcSp84 of Cytospora chrysosperma Localizes to the Plant Nucleus to Trigger Plant Immunity
}

\author{
Zhiye $X u^{1,2}$, Dianguang Xiong ${ }^{1,2, *} \mathbb{C}$, Zhu Han ${ }^{1,2}$ and Chengming Tian ${ }^{1,2, *}$ \\ 1 Beijing Key Laboratory for Forest Pest Control, Beijing Forestry University, Beijing 100083, China; \\ xuzhiye987@163.com (Z.X.); hanandzhu@163.com (Z.H.) \\ 2 The Key Laboratory for Silviculture and Conservation of Ministry of Education, College of Forestry, \\ Beijing Forestry University, Beijing 100083, China \\ * Correspondence: xiongdianguang@126.com (D.X.); chengmt@bjfu.edu.cn (C.T.)
}

check for updates

Citation: Xu, Z.; Xiong, D.; Han, Z.; Tian, C. A Putative Effector CcSp84 of Cytospora chrysosperma Localizes to the Plant Nucleus to Trigger Plant Immunity. Int. J. Mol. Sci. 2022, 23, 1614. https://doi.org/10.3390/ ijms23031614

Academic Editor: Artemio Mendoza

Received: 30 December 2021

Accepted: 28 January 2022

Published: 30 January 2022

Publisher's Note: MDPI stays neutral with regard to jurisdictional claims in published maps and institutional affiliations.

Copyright: (C) 2022 by the authors. Licensee MDPI, Basel, Switzerland. This article is an open access article distributed under the terms and conditions of the Creative Commons Attribution (CC BY) license (https:// creativecommons.org/licenses/by/ $4.0 /)$.

\begin{abstract}
Cytospora chrysosperma is the main causal agent of poplar canker disease in China, especially in some areas with poor site conditions. Pathogens secrete a large number of effectors to interfere the plant immunity and promote their infection and colonization. Nevertheless, the roles of effectors in C. chrysosperma remain poorly understood. In this study, we identified and functionally characterized a candidate effector CcSp84 from C. chrysosperma, which contained a nuclear localization signal motif at the C-terminal and was highly induced during infection stages. Transient expression of CcSp84 in Nicotiana benthamiana leaves could trigger cell death. Additionally, deletion of CcSp84 significantly reduced fungal virulence to the polar twigs, while no obvious defects were observed in fungal growth and sensitivity to $\mathrm{H}_{2} \mathrm{O}_{2}$. Confocal microscopy revealed that $\mathrm{CcSp} 84$ labeled with a green fluorescent protein (GFP) was mainly accumulated in the plant nucleus. Further analysis revealed that the plant nucleus localization of CcSp84 was necessary to trigger plant immune responses, including ROS accumulation, callose deposition, and induced expression of jasmonic acid and ethylene defenserelated genes. Collectively, our results suggest that $\mathrm{CcSp} 84$ is a virulence-related effector, and plant nucleus localization is required for its functions.
\end{abstract}

Keywords: Cytospora chrysosperma; effector; pathogenicity; subcellular localization; plant immunity

\section{Introduction}

Cytospora chrysosperma is a necrotrophic fungal pathogen that causes stem canker in a broad range of hosts $[1,2]$. In nature, the spores of $C$. chrysosperma germinate and produce the hyphae to invade the host plants with poor conditions through the wound. The hyphae then infect the phloem and primary xylem in the horizontal and vertical directions and finally extend to the pith $[3,4]$. With the spread of this pathogen, the canker disease has become more widespread and difficult to control, especially in China [4,5]. Currently, studies about C. chrysosperma are focused on the taxonomy, genetic diversity, histopathology, and gene function survey $[3,4,6,7]$, while the molecular mechanism studies were few reported. For example, the mitogen-activated protein kinase (CcPmk1) and oxalic acid metabolism were required for the growth and virulence of $C$. chrysosperma $[5,8,9]$. The virulence effector CcCAP1, a member of the CAP superfamily (cysteine-rich secretory protein, antigen 5, and pathogenesis-related 1), from C. chrysosperma mainly localized to plant nucleus to suppress plants immune responses [2]. Additionally, the transcription factor CcSge1, belonging to the Gti1/Pac2 family, was essential to the hyphal radial growth, conidiation, and fungal virulence. Intriguingly, both $\mathrm{CcSge} 1$ and $\mathrm{CcPmk} 1$ positively regulated the expression of the putative effector gene CcCAP1 in C. chrysosperma [10]. However, the molecular interaction mechanism between $C$. chrysosperma and plants is still unclear.

Plants and phytopathogens have coevolved for a long time and have established a two-layer interaction system: pathogen-associated molecular patterns (PAMPs)-triggered 
immunity (PTI) and effector-triggered immunity (ETI) [11-13]. Plant plasma membranelocalized pattern recognition receptors (PRRs) can sense pathogens through recognition of the highly conserved PAMPs, such as flagellin, chitin, and glucans, to activate the PTI $[14,15]$. Additionally, plant intracellular nucleotide-binding and leucine-rich repeat receptors (NLRs) can detect cytoplasmic effectors to induce the ETI responses, including the activation of a series of downstream defense-related responses [16-19].

Recently, studies have shown that ETI can enhance the PTI defense responses [20]. Phytopathogens secrete a series of effectors to disturb the plant defense responses and promote colonization, while some of them can trigger plant defense responses, known as avirulent protein. More and more evidence has demonstrated that effectors could locate to the plant nucleus to carry out their functions. HopA1 $1_{\mathrm{Pss} 61}$ from Pseudomonas syringae is co-localized with Arabidopsis R protein RPS6 in the nucleus and cytoplasm, but the nucleus localization of HopA1 $1_{\mathrm{Ps} 61}$ is required to induce cell death [21]. Pop2, an avirulence type III effector from Raistonia solanacearum, can be recognized by the RRS1-R in the nucleus, which will grant broad-spectrum resistance to several different strains of Raistonia solanacearum. The Verticillium-specific secreted protein VdSCP7 localized to the plant nucleus to trigger plant immunity, and its nuclear localization was required to activate plant defense responses [22]. Similar results were also reported in other effectors from different phytopathogens. In Phytophthora infestans, RXLR effector Pi04314 could be active in the host nucleus to reduce the expression of jasmonic and salicylic acid-related defense genes and induce the relocation of three host protein phosphatase 1 catalytic isoforms from the nucleolus to nucleoplasm during infection to enhance leaf colonization [23]. PvAVH53 located in the plant nucleus and could interact with tobacco importin- $\alpha$ genes, NbImp- $\alpha 1$ and NbImp- $\alpha 2$, which were essential to induce cell death in N. benthamiana leaves. The silencing of importin- $\alpha$ s genes resulted in increased susceptibility to the oomycete pathogen Phytophthora capsici in N. benthamiana [24].

In this study, two candidate effectors (CcSp31 and CcSp84), both containing a nuclear localization signal motif at the C-terminal, were functionally characterized in C. chrysosperma. The results showed that $\mathrm{CCSp} 84$ was required for the fungal pathogenicity and could trigger plant cell death in N. benthamiana leaves, while CcSp31 deletion mutants displayed no obvious defects compared to the wild type, and it could not trigger cell death or suppress the INF1-induced cell death in N. benthamiana. Further analyses suggest that the plant nucleus localization of CcSp84 was important for its activation functions.

\section{Results}

\subsection{Two NLS Motif Containing Candidate Effectors Were Induced during Infection Processes}

Pathogens secrete a series of effectors into the host cytoplasm and apoplast to disturb the host immunity [25]. As expected, numerous effector candidates were predicted in C. chrysosperma according to typical criteria, such as small in length, containing a signal peptide, no other transmembrane domains, and being rich in cysteine residues $[26,27]$ (data not shown). Among them, two candidate effector genes, both containing a nuclear localization signal at the C-terminal, were selected, named CcSp31 and CcSp84, in this study. CcSp84 contained a glycoside hydrolase 61 domain, while no annotated domain was found in CcSp31 (Figure 1A, Table 1). Remarkably, both of them were significantly up-regulated during the infection stages, and they both showed the highest expression levels at 3 dpi (Figure 1B), indicating their involvements in the fungal pathogenicity. 
A
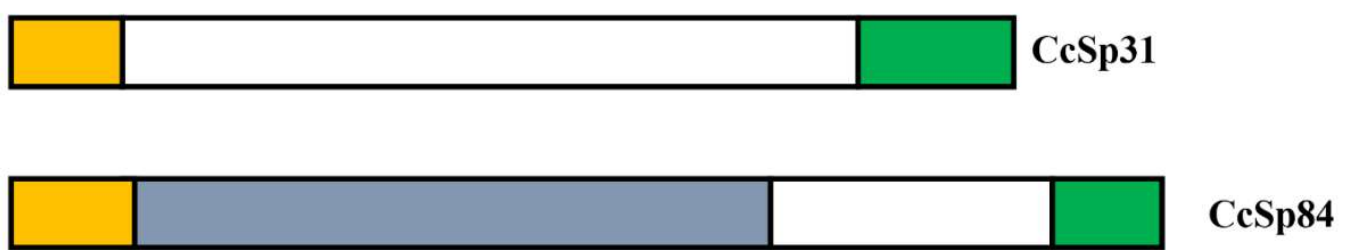

$\square$ Signal Peptide $\square$ Glycoside hydrolase family $61 \square$ Nuclear Location Signal

B
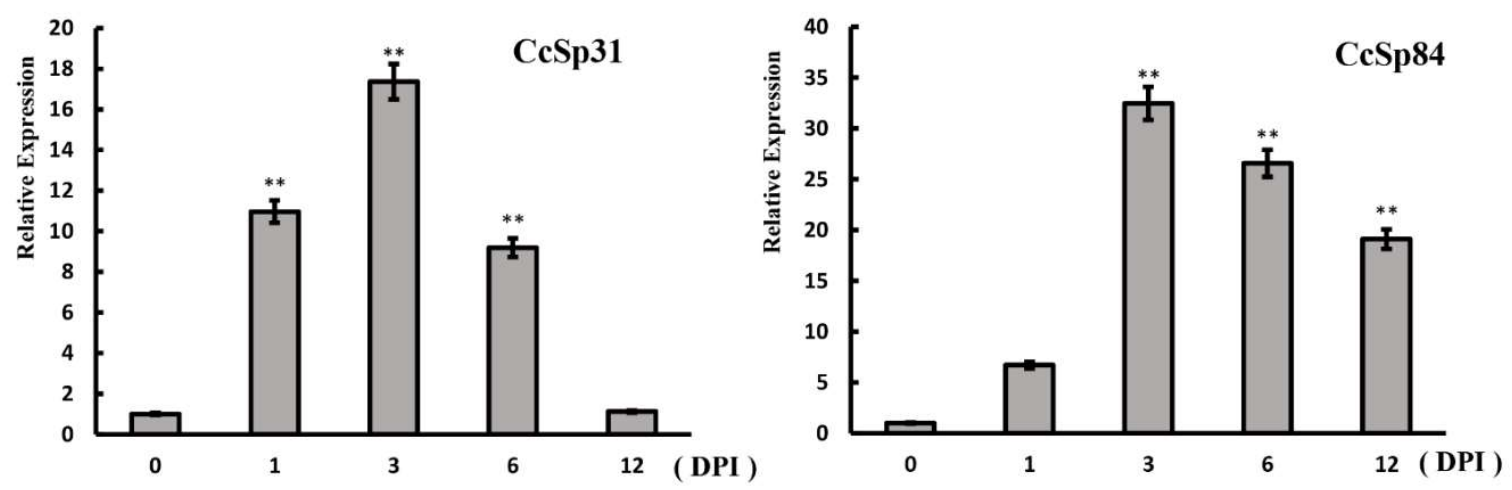

Figure 1. $C c S p 31$ and $C c S p 84$ were significantly up-regulated during infection stages. (A) Schematic diagram of candidate effectors CcSp31 and CcSp84. (B) Relative expression levels of CcSp31 and CcSp84 during infection processes. The expression levels were monitored using reverse transcriptionquantitative polymerase chain reaction (qRT-PCR). RNA was extracted at 0, 1, 3, 6, and 12 days post-inoculation (DPI) on the poplar twigs with Cytospora chrysosperma. The experiments were performed in triplicate. ${ }^{* *} p<0.01$.

Table 1. Basic information of selected candidate effectors CcSp31 and CcSp84.

\begin{tabular}{ccc}
\hline Gene Name & CcSp31 & CcSp84 \\
\hline Gene ID & GME4592_g & GME8128_g \\
Signal Peptide & $\mathrm{Y}$ & $\mathrm{Y}$ \\
$\begin{array}{c}\text { Protein length } \\
\text { (SP truncated) }\end{array}$ & $277(260)$ & $315(293)$ \\
$\begin{array}{c}\text { Cysteines number } \\
\text { homologous gene } \\
\text { NLS sequence }\end{array}$ & 5 & 3 \\
\hline
\end{tabular}

\subsection{CcSp84 Induced Cell Death in N. benthamiana Leaves}

To investigate the function of C CSp31 and CcSp84 during the interaction processes, CcSp31 and CcSp84 were cloned into the binary vector pGR106 without signal peptide, respectively [28], and then transiently expressed in $N$. benthamiana leaves by using the Agrobacterium tumefaciens-mediated system. After 3 days of infiltration, CcSp31 neither induced cell death nor suppressed the INF1-induced cell death in N. benthamiana leaves (Figures 2A and S1). Remarkably, CcSp84 could induce cell death in N. benthamiana leaves as the INF1 did, but it could not suppress the INF1-induced cell death in N. benthamiana leaves (Figure 2B), indicating that CcSp84 acted as an elicitor and could induce cell death in N. benthamiana leaves. 
A

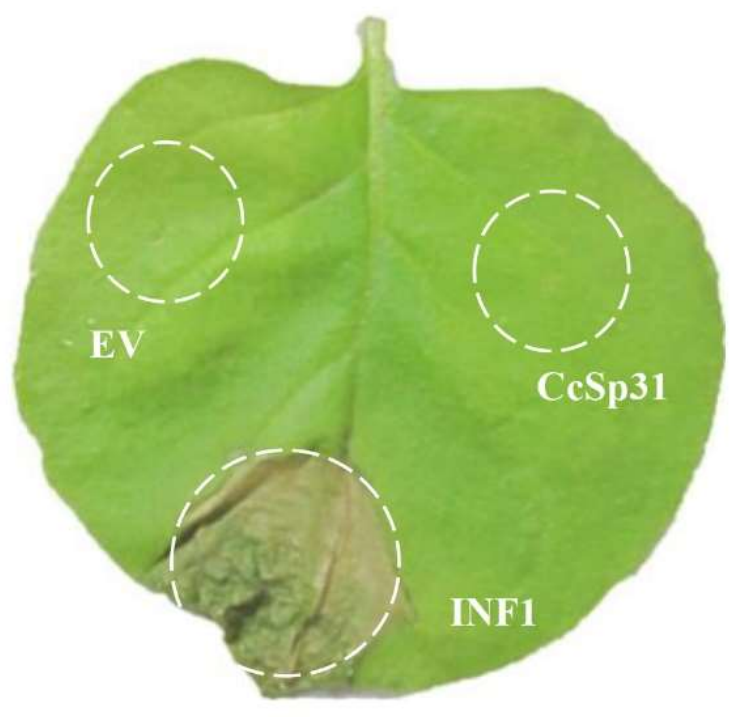

B

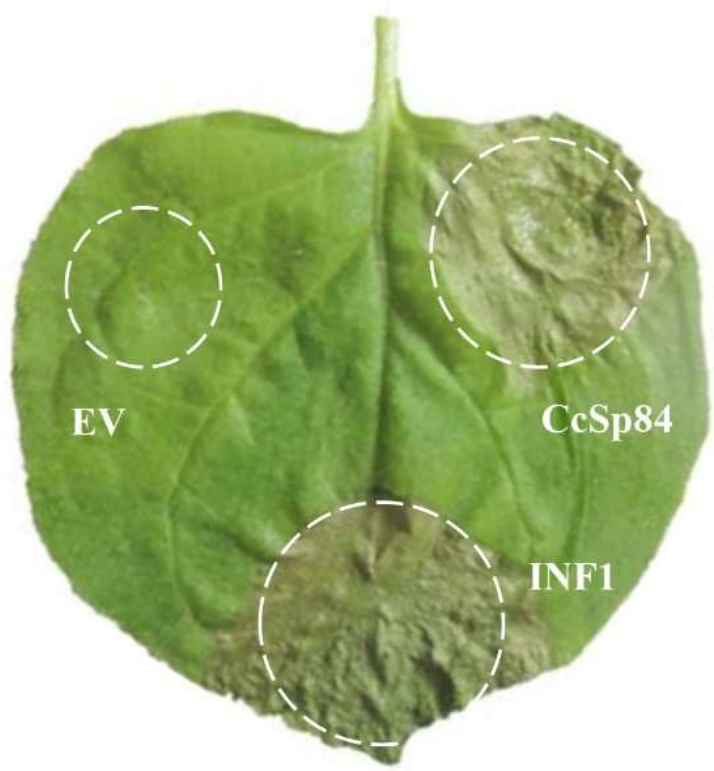

Figure 2. CcSp31 and CcSp84 were transiently expressed into $N$. benthamiana leaves by agro-infiltration. (A) pGR106 (Empty vector, EV), CcSp31-pGR106, and INF1-pGR106 were infiltrated in N. benthamiana leaves, respectively. (B) EV, CcSp84-pGR106, and INF1-pGR106 were infiltrated in N. benthamiana leaves, respectively. The pictures were taken at $3 \mathrm{dpi}$. The data represented have three independent biological repeats.

\subsection{CcSp31 and CcSp84 Were Not Required for Fungal Growth and the Resistance to $\mathrm{H}_{2} \mathrm{O}_{2}$}

To further determine the potential function of CcSp31 and CcSp84 in C. chrysosperma, the gene deletion fragments were constructed through the split-marker method (Supplementary Figure S2). Two deletion mutants of CcSp31 ( $\Delta C c S p 31-31$ and $\Delta C c S p 31-38$ ) and two deletion mutants of $C c S p 84(\Delta C c S p 84-5$ and $\Delta C c S p 84-10)$ were successfully obtained by using the PCR screening and Southern blot analyses (Figure 3A-C). The complementary strains were generated in a similar way (Supplementary Figure S1), named $\Delta C c S p 31 / C$ and $\Delta C c S p 84 / C$. The mycelial plugs of normal wild type (WT), $\Delta C c S p 31-31$, $\Delta C c S p 31-38, \Delta C c S p 84-5, \Delta C c S p 84-10$, and their complementary strains were inoculated in the PDA medium. The results showed that no significant differences were found among all the strains (Figure 4A,B), indicating that CcSp31 and CcSp84 were not required for the fungal growth.

To examine the resistance of $C c S p 31$ and $C c S p 84$ deletion mutants to $\mathrm{H}_{2} \mathrm{O}_{2}$, conidial suspensions of WT, $\Delta C c S p 31, \Delta C c S p 84, \Delta C c S p 31 / C$, and $\Delta C c S p 84 / C\left(1 \times 10^{6}\right.$ conidiospore $\left./ \mathrm{mL}\right)$ were added into homogeneously melted and warmed PDA medium, then a filter paper with $6 \%$ and $8 \% \mathrm{H}_{2} \mathrm{O}_{2}$ was placed in the middle of the plates, as described previously (Wang et al., 2018). No distinguish differences in responses to the $\mathrm{H}_{2} \mathrm{O}_{2}$ stress were observed among the CcSp31 and CcSp84 deletion mutants, complementary strains, and the WT (Figure 4C,D). The data illustrate that CcSp31 and CcSp84 were dispensable for fungal growth and the resistance to $\mathrm{H}_{2} \mathrm{O}_{2}$.

\subsection{CcSp84 Was Essential for Fungal Pathogenicity}

To calculate the putative roles of CcSp31 and CcSp84 in the fungal pathogenicity, we scalded the Populus euramericana branches and then inoculated them with mycelial plugs of WT, $\Delta C c S p 31$ and $\Delta C c S p 84$, and their complementary strains. As shown in Figure 5A, $\mathrm{B}$, the lesion areas were significantly reduced in $\Delta C c S p 84-5$, and $\Delta C c S p 84-10$ inoculated branches, compared to that in the WT and complementary strains, while comparable lesion areas were observed in CcSp31 deletion mutants and WT inoculated branches (Figure 5C,D). The above results suggest that $\mathrm{CcSp} 84$ is a pathogenicity-related elicitor in C. chrysosperma; therefore, $\mathrm{C} c \mathrm{Sp} 84$ was selected for further analyses. 
A

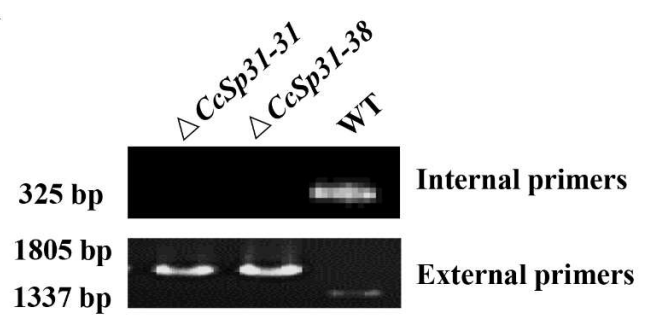

B

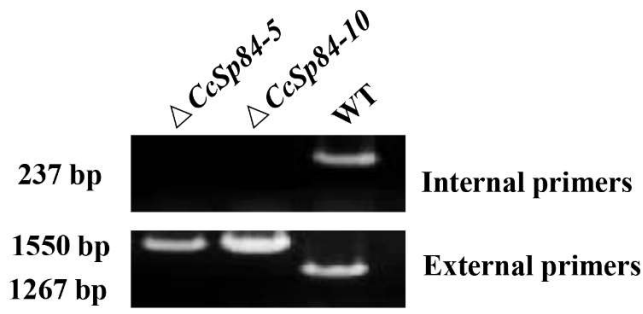

C

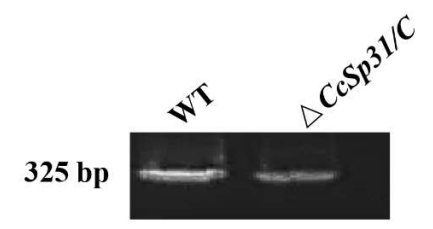

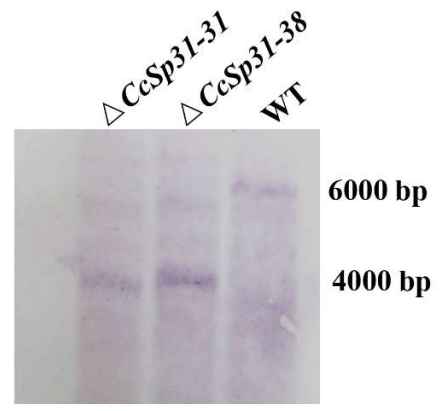

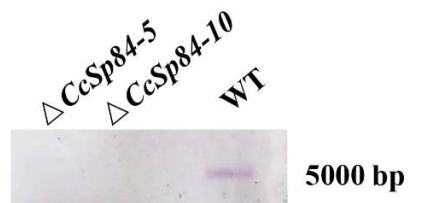

bp

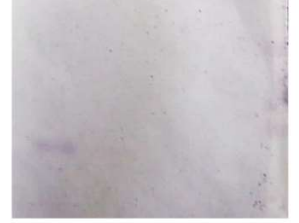

$1000 \mathrm{bp}$

Figure 3. Screening for the CcSp31 and CcSp84 deletion mutants. (A,B) The CcSp31 and CcSp84 deletion mutants were confirmed by PCR with internal primers and external primers and Southern blot analysis. (C) The complementary strains were verified by PCR with internal primers.

A
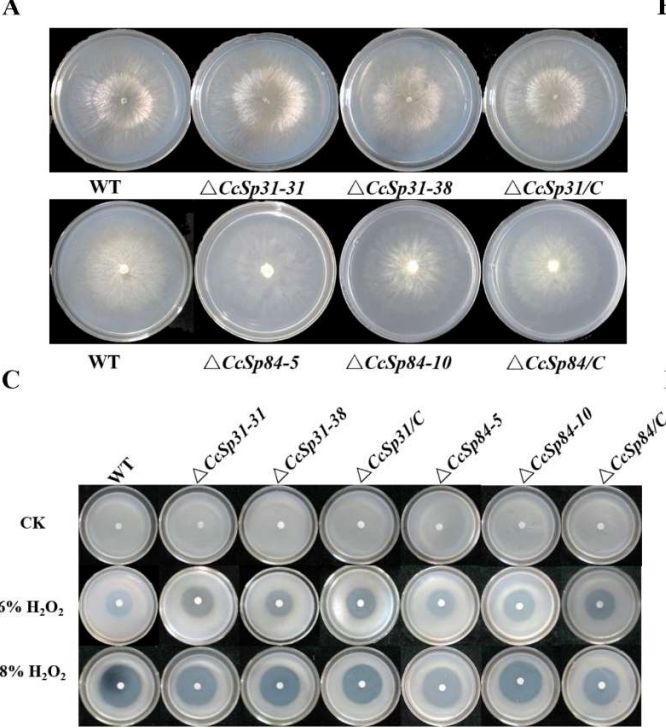

B
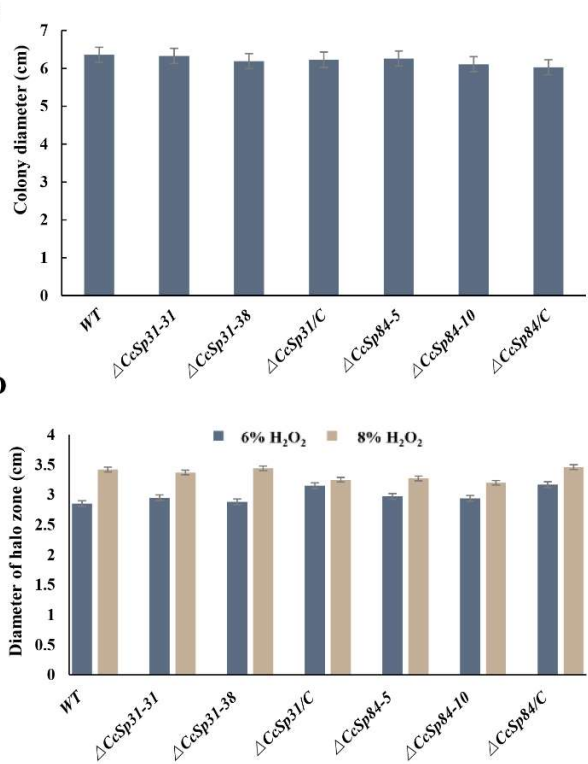

Figure 4. $C c S p 31$ and $C c S p 84$ were not required for the fungal growth and resistance to $\mathrm{H}_{2} \mathrm{O}_{2}$. (A) Colony morphologies of wild-type (WT), CcSp31 and CcSp84 deletion mutants, and complemented strains after $48 \mathrm{~h}$ of growth on PDA plates. (B) Colony diameters of the strains on PDA plates. (C) Colony morphology of wild-type, CcSp31 and CcSp84 deletion mutants, and each complemented strains treated with different concentrations of $\mathrm{H}_{2} \mathrm{O}_{2}$. The pictures were taken at 60 hpi. (D) Diameter of halo zones of the strains on PDA plates supplemented with $\mathrm{H}_{2} \mathrm{O}_{2}$. 
A

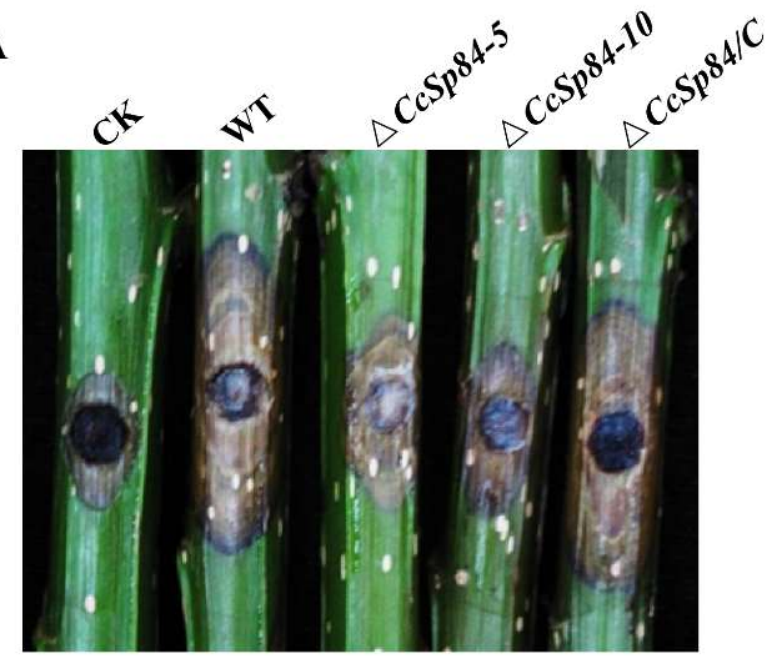

C

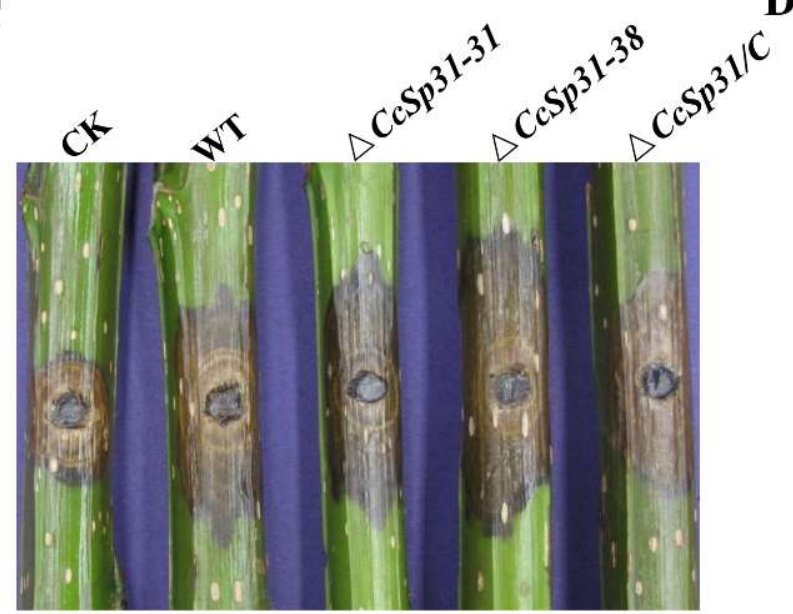

B
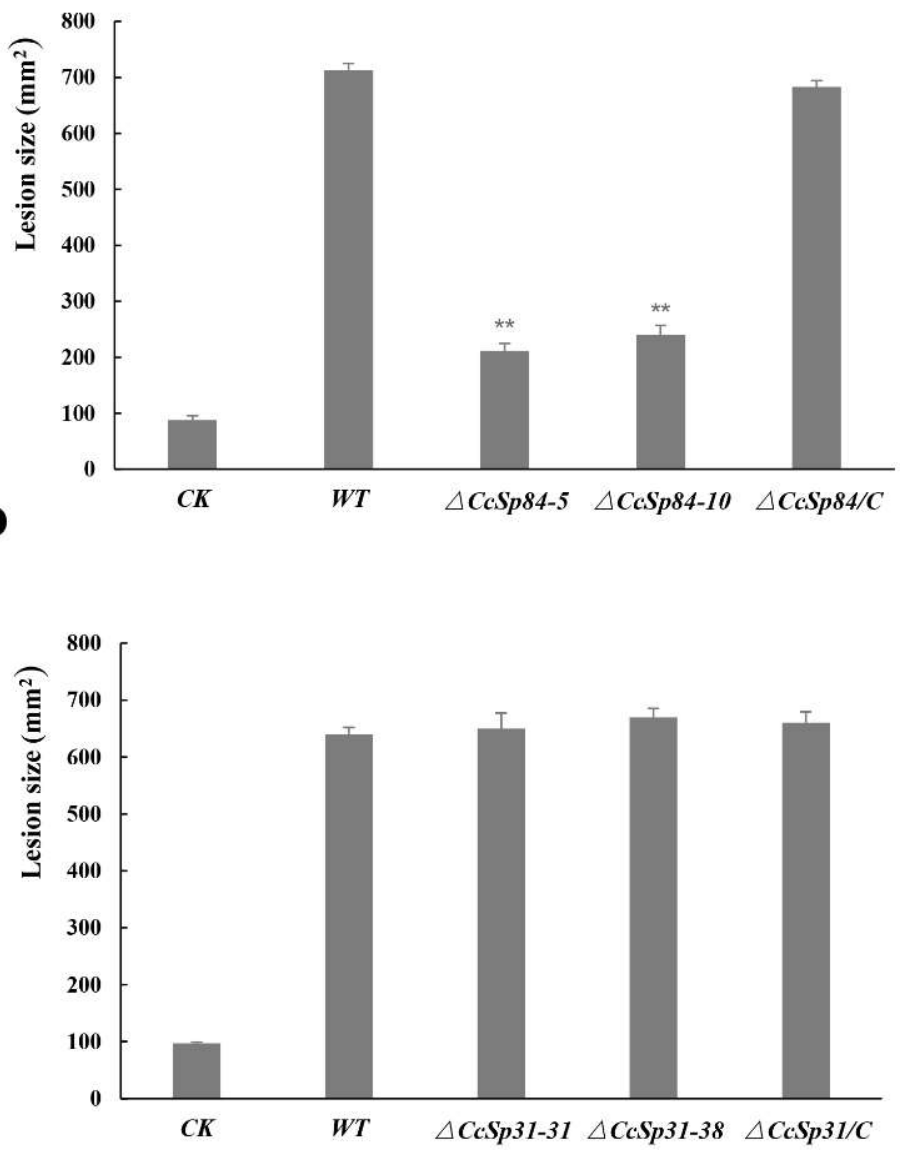

Figure 5. The CcSp84 deletion mutants reduce the pathogenicity of C. chrysosperma. (A,B) Inoculated poplar branches with wild-type, CcSp84 deletion mutants, and complemented strains, the lesion areas were measured at $4 \mathrm{dpi}$. (C,D) Inoculated poplar branches with wild-type, CcSp31 deletion mutants, and complemented strains, the lesion areas were measured at $4 \mathrm{dpi}$. The asterisks on the bars indicate a significant difference from the wild-type strain $(* * p<0.01)$. The experiments were repeated three times.

\subsection{The Nuclear Localization of CcSp84 Was Required to Induce Plant Defense Responses}

As shown in Figure 1A, CcSp84 contains a predicted NLS motif at C-terminal. In order to clarify the subcellular localization of CcSp84 in the plant cell and the functions of the NLS motif, four recombinant plasmids were constructed, including CcSp84-GFP2, CcSp84_nls (deletion of NLS sequences), CcSp84_NES (adding the NES sequences at the C-terminal), and CcSp84_nls_NES (deletion of NLS sequences and adding the NES sequences at the C-terminal) (Figure 6A). Then, these recombinant plasmids of CcSp84 were introduced into the A. tumefaciens GV3101 and transiently expressed in N. benthamiana leaves. Confocal microscopy results showed that the fluorescence of CcSp84-GFP was mainly localized in the plant nucleus, while the fluorescence signal of CcSp84_nls and CcSp84_NES could be observed in both plant nucleus and cytoplasm. Additionally, the fluorescence signal of CcSp84_nls_NES was only detectable in the plant cytoplasm but not the plant nucleus (Figure 6B). These results indicated that CcSp 84 was mainly located in the plant nucleus and the NLS motif was important for its plant nucleus localization. 
A

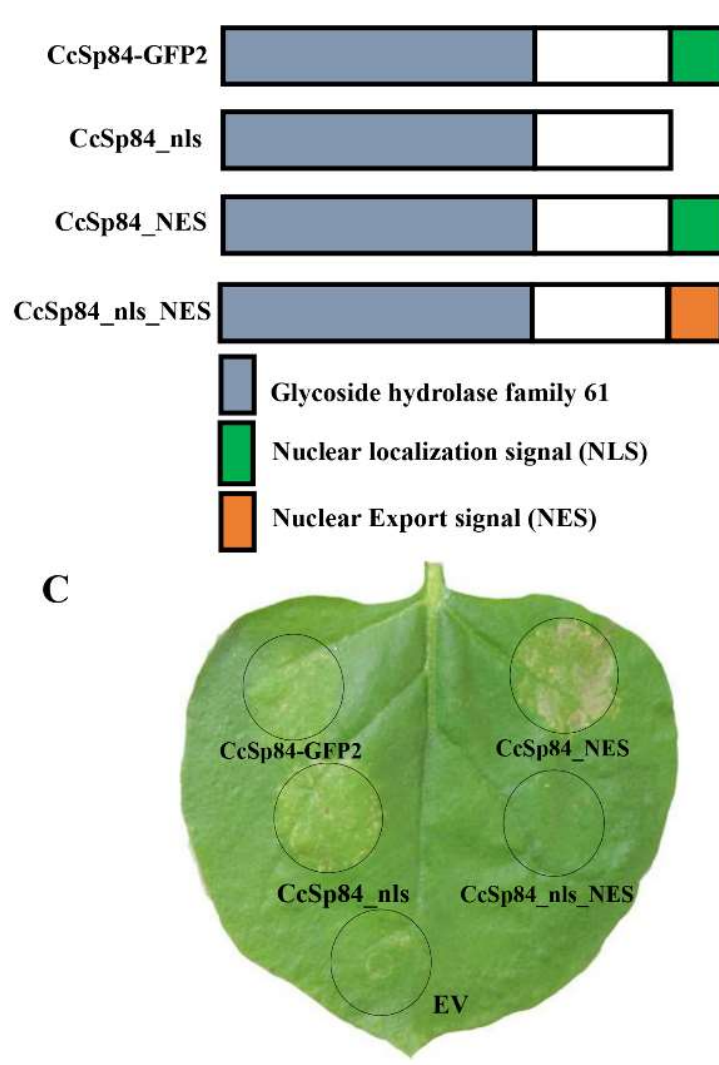

B

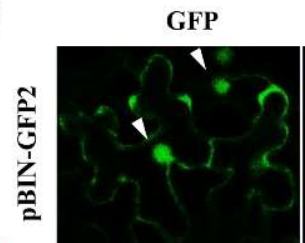

DAPI
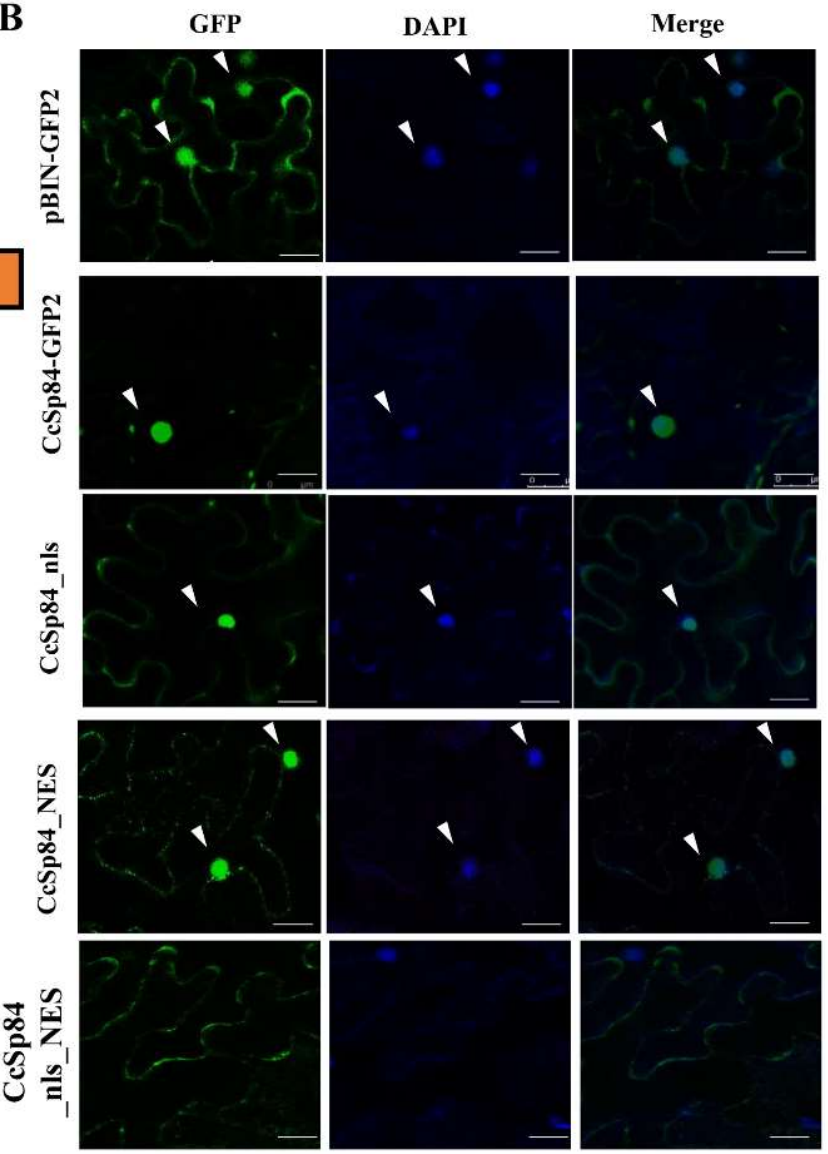

D

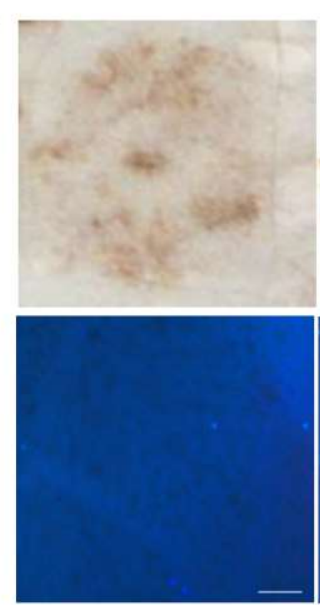

pBIN-GFP2

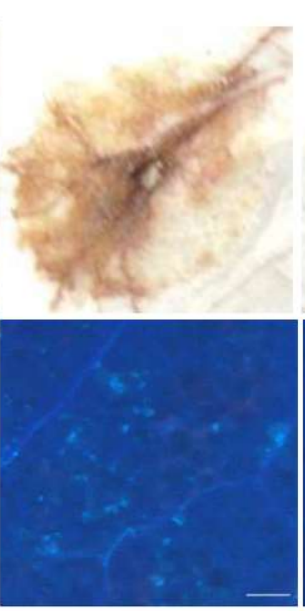

CeSp84-GFP2

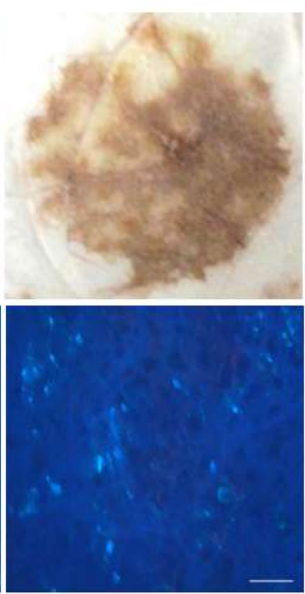

CcSp84_nls

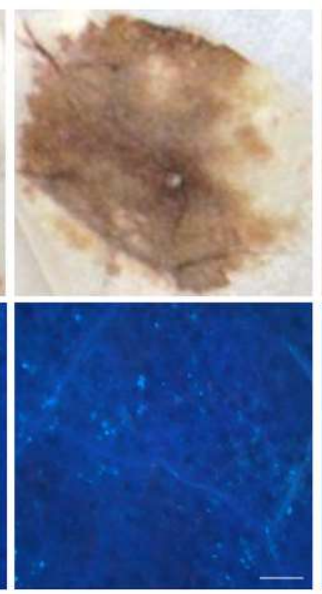

CcSp84-NES

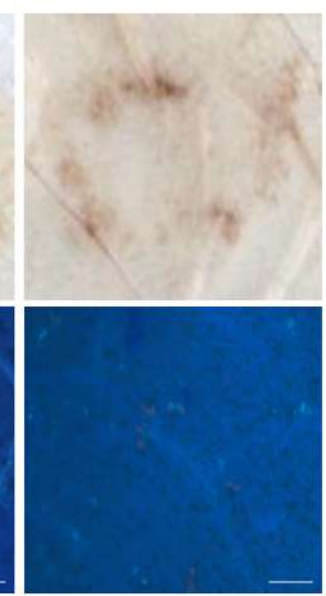

CcSp84_nls_NES

Figure 6. CcSp84 located in the plant nucleus to trigger plant defense responses. (A) Diagram structure of different mutant types of CcSp84 fused to GFP. (B) Subcellular localization of GFP-tagged CcSp84 mutant types when transiently expressed in N. benthamiana. Confocal Microscope SP8 with wavelengths of 488 and $406 \mathrm{~nm}$ for GFP and DAPI, respectively. The pictures were photographed at 3 dpi. Bars, $5 \mu \mathrm{m}$. (C) Different mutant types of CcSp84 were transiently expressed in N. benthamiana. (D) The detection of ROS and callose deposition in N. benthamiana leaves at $24 \mathrm{~h}$ post-inoculation. Bars, $200 \mu \mathrm{m}$.

To analyze the relationship between the C $\operatorname{cSp} 84$ localization and its function, the response of $N$. benthamiana leaves infiltrated the recombinant $A$. tumefaciens containing different mutants of CcSp84 plasmids was observed. The results showed that CcSp84_GFP2, CcSp84_nls, and CcSp84_NES could induce different levels of plant cell death after three 
days of inoculation, whereas CcSp84_nls_NES was unable to induce cell death (Figure 6C). Additionally, obvious ROS accumulation and callosum deposition were detected in the $N$. benthamiana leaves infiltrated with CcSp84_GFP2, CcSp84_nls, and CcSp84_NES at $24 \mathrm{~h}$ post-inoculation, while comparable results were found between control treatment and CcSp84_nls_NES (Figure 6D). Together, these results demonstrate that the nuclear localization of CcSp84 is essential to trigger plant immunity.

\subsection{Expression of CcSp84 Could Induce the Expression of Defense-Related Genes in N. benthamiana}

Previous studies have proved the synergism between jasmonic acid (JA) and ethylene (ET) signaling pathways, which are required for the resistance against necrotrophic pathogens through the regulation of plant defense-related genes [22,29,30]. To assess whether CcSp84 could affect the expression of defense genes in the JA and ET signaling pathways, the expression of marker genes involved in JA and ET signaling pathways were calculated, including NbPR4 (Niben101Scf12045g06025.1), NbLOX (Niben101Scf01434g03006.1), and NbERF1 (Niben101Scf00454g04003.1) [31-33]. As shown in Figure 7, the expression of NbPR4, NbLOX, and NbERF1 was significantly induced in the $N$. benthamiana leaves transiently expressed CcSp84,CcSp84_nls, and CcSp84_NES recombinant strains compared with control, which indicated that transiently expressed CcSp84 in N. benthamiana would activate the JA and ET-related signal pathway to trigger plant immune responses.
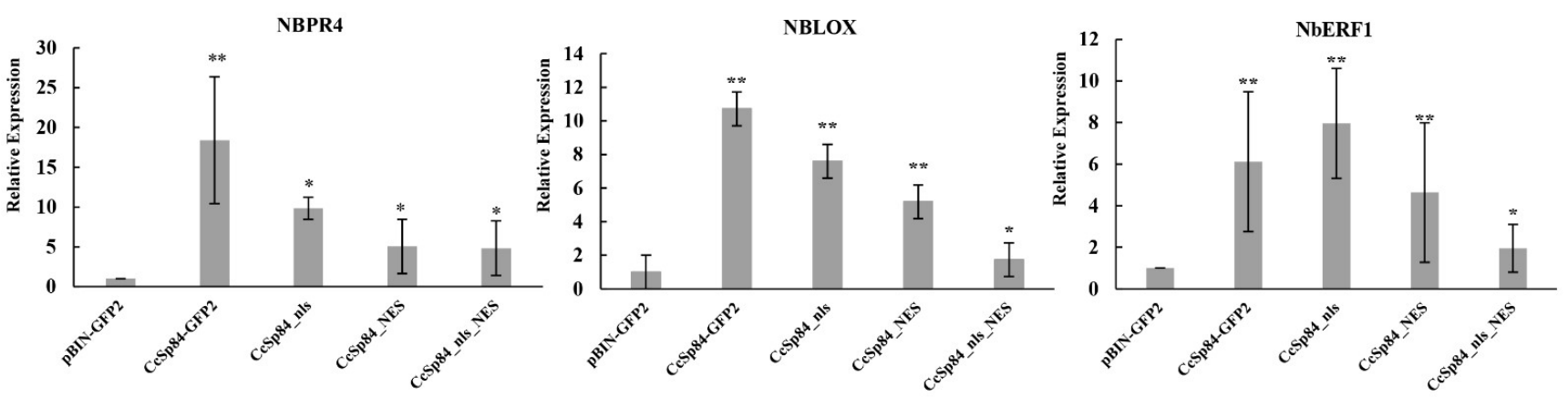

Figure 7. Relative expression levels of defense-related genes involved in JA and ET signal pathways in N. benthamiana. The marker genes of JA and ET-dependent defense responses were significantly up-regulated after being transiently expressed the CcSp84 in N. benthamiana. The experiments were performed three times. ${ }^{*} p<0.05,{ }^{* *} p<0.01$.

\section{Discussion}

In plant-pathogen interactions, the plant nucleus is a core battlefield, and various interactions were involved. Effectors and receptors play an important role in determining the overall outcome of infection [22,33,34]. In this study, we identified and functionally characterized a small, cysteine-rich secreted protein, $C c S p 84$, which acted as a key virulencerelated candidate effector of C. chrysosperma. CcSp84 was strongly induced during infection stages and was required for the pathogenicity of $C$. chrysosperma. Transient expression of CcSp84 on the N. benthamiana leaves could trigger plant immune responses, and its nuclear localization was essential to activate the plant defense responses.

It is well known that phytopathogens secret a large number of effectors to the extracellular matrix during the infection process. Some of them are located at the extracellular matrix, while some of them are located at the cytoplasm [35,36]. The different locations of the effectors may represent various regulation manners. For example, VdEG1 and VdEG3 from Verticillium dahliae, which belonged to the Glycoside Hydrolase Family (GH12), were identified as apoplastic elicitors and mediated the plasma membrane-localized receptor BAK1 to regulate the host immunity [37]. PstGSRE1 from Puccinia striiformis f. sp. tritici (Pst) targeted the ROS-associated transcription factor TaLOL2 by disrupting the nuclear localization of TaLOL2 and suppressing the ROS-mediated cell death induced by TaLOL2 [38]. 
Additionally, a putative effector VdSCP7 from Verticillium dahliae contained an NLS motif, which was also located in the plant nucleus and modulated plant immunity. Here, CcSp84 contained a predicted NLS motif (Figure 1A) and was localized to the plant nucleus to induce plant immune responses (Figure 6C). The results suggest that the NLS motif may lead to the nucleus location of effectors in the plant cell. More importantly, many studies have reported that the correct localization of effectors to specific subcellular compartments is essential for their functions. For example, transiently expressed full-length PsXEG1 (including signal peptide) or PR1-PsXEG1 ${ }^{20-241}$, an apoplast effector from Phytophthora sojae, could trigger cell death, while transiently expressed PsXEG1 ${ }^{20-241}$ (without signal peptide) did not trigger cell death in N. benthamiana, indicating that the PsXEG1 must target the apoplast to achieve its functions [39]. Similar results were also found in the XEG1 homologs from Botrytis cinerea and $V$. dahliae [37,40]. In this study, transiently expressed CcSp84 in $N$. benthamiana leaves could induce cell death, and fluorescence observation found that CcSp84 is located in the plant nucleus. Additionally, CcSp84_NES was localized to the plant cytoplasm and nucleus, indicating that the nuclear export signal (NES) sequence can partly induce the exportation of $\mathrm{C} C \mathrm{Sp} 84$ from the nucleus to cytoplasm. However, when deleting the NLS sequence and adding an NES sequence to the CcSp84, the GFP signal was almost abolished in the nuclei in CCSp84_nls_NES expressing leaves. Similar results were also found in the plant nuclear localization effector VdSCP7 from Verticillium dahliae, which also contains an NLS sequence [22]. Therefore, we speculated that when the protein contains both NLS and NES sequences, its localization may depend on the functional ability of these two motifs. Intriguingly, its elicitor activity was compromised when it manually exported CcSp84 to the cytoplasm region, indicating that the plant nuclear localization is essential for $C c S p 84$ to trigger plant immune responses.

Generally, the expression of secreted effectors would be induced during the infection or colonization processes, and several of them may be stage-specific [41]. Additionally, numerous effectors were reported to be involved in pathogenicity because of their crucial roles during the interactions between pathogens and host plants $[13,36,42,43]$. In stripe rust fungus, a haustorium-specific effector, Pst_12806, was significantly up-regulated during infection stages, and it could suppress the plant basal immunity by reducing the callose deposition and the expression of defense-related genes [44]. However, many of them did not show obvious defects in pathogenicity with single deletion of the effector genes, which was possibly due to the functional redundancy. For example, the LysM-type effector from Zymoseptoria tritici, Mgx1LysM, was essential for the lesion's extension through binding to the chitin and then suppressed the chitin-induced ROS burst, and was able to protect fungal hyphae against host chitinase hydrolysis [45]. In this study, CcSp84 was significantly induced during the infection stages, and transient expression of CcSp 84 in $N$. benthamiana would cause cell death and activate the plant defense responses, including the burst of ROS, deposits of callosum, and induced expression of defense-related genes. Importantly, CcSp84 was required for fungal pathogenicity. On the other hand, CcSp31 was also significantly up-regulated during the infection stages, but the deletion of CcSp31 did not exhibit defects in fungal pathogenicity compared to the wild type. Therefore, we speculated that the functional redundancy of effectors and the dependence of expression pattern during infection stages may lead to the non-visualization of fungal virulence.

Increasing evidence is available for the positive roles of plant JA-mediated signaling against biotrophic and necrotrophic pathogens [30,46-48]. Pseudomonas syringae releases type III effector HopX1, a cysteine protease, which has been reported to interact with the ZIM domain of jasmonate family members and degrade them, resulting in plant susceptibility [49]. The AvrB effector RIN4 activated the plasma membrane ATPase AHA1 to cause an alteration in membrane potential, which increased the interaction between COI1 and JAZ, ultimately leading to degradation of the JA-related proteins [50]. Here, we investigated the expression pattern of three marker genes related to the immune-related signaling pathways: JA and ET, and the results demonstrated that $C c S p 84$ could positively regulate the JA and ET signaling pathway. 
Collectively, we functionally characterized two putative NLS motifs containing effectors, CcSp31 and CcSp84, from C. chrysosperma. The CcSp31 deletion mutants displayed no defects in the vegetative growth, stress response, and virulence compared to the wild-type, while CcSp84 was critical for fungal pathogenicity, which could also induce cell death and immune responses in $N$. benthamiana. Further analysis revealed that the plant nucleus localization of $C c S p 84$ was essential to trigger the plant defense responses.

\section{Material and Methods}

\subsection{Fungal Strains and Plants Growth Conditions}

The wild-type (WT) strain (CFCC_89981) of C. chrysosperma was preserved in the forest pathology laboratory in Beijing Forestry University (Strain No: G-YS-11-C1). The WT, deletion, and complementation mutants were all cultured in a potato dextrose agar (PDA) medium (200 g potato filtrate, $20 \mathrm{~g}$ glucose, $15 \mathrm{~g}$ agar per liter) at $25^{\circ} \mathrm{C}$ in the dark. Conidia were incubated in a yeast extract peptone dextrose (YEPD) medium ( $3 \mathrm{~g}$ yeast extract, $10 \mathrm{~g}$ bacto peptone, and $20 \mathrm{~g}$ glucose per liter) for the preparation of protoplasts and transformants. Furthermore, $\mathrm{TB}_{3}$ medium ( $3 \mathrm{~g}$ yeast extract, $3 \mathrm{~g}$ casamino acids, $200 \mathrm{~g}$ sucrose, $7 \mathrm{~g}$ agar per liter) with $30 \mu \mathrm{g} / \mathrm{mL}$ hygromycin, was used to select transformants. The cultures of $C$. chrysosperma were grown in a liquid potato dextrose broth (PDB) medium (200 g potato filtrate, $20 \mathrm{~g}$ glucose per liter) at $25{ }^{\circ} \mathrm{C}$, shaking at $250 \mathrm{rpm}$ for DNA or RNA extraction. E. coli DH5 $\alpha$ cultured in lysogeny broth (LB) at $37^{\circ} \mathrm{C}$ were used for plasmid construction. Agrobacterium strain GV3101 was cultured at $30^{\circ} \mathrm{C}$ and used for Agrobacterium-mediated transient expression in N. benthamiana leaves. N. benthamiana was grown in a greenhouse at $25^{\circ} \mathrm{C}$ and $70 \%$ relative humidity with a $16 / 8 \mathrm{~h}$ of day/night light for about four weeks.

\subsection{Bioinformatics Analysis}

The putative effectors of $C$. chrysosperma could be identified by several prominent features, such as the signal peptide, cysteine-rich domain, small length, and no additional transmembrane structure [51]. In this research, the SignalP 5.0 Server (http:/ / www.cbs. dtu.dk/services/SignalP-5.0/, accessed on 27 August 2019) and TMHMM Server 2.0 (http:/ /www.cbs.dtu.dk/services/TMHMM/, accessed on 27 August 2019) were used to search the signal peptide and the domain of transmembrane structure. The Interpro (http:/ / www.ebi.ac.uk/interpro/, accessed on 28 August 2019), Smart Page (http:/ / smart. embl-heidelberg.de/, accessed on 28 August 2019) and cNLS Mapper (http:/ /nls-mapper. iab.keio.ac.jp/cgi-bin/NLS_Mapper_form.cgi, accessed on 28 August 2019) were used to detect the gene family, functional domains, and nuclear localization signal motif.

\subsection{Gene Knockout and Complementation}

The target genes, CcSp31 and CcSp84, were knocked out by the split-marker method [52,53]. According to the method, the upstream and downstream flanking sequences, about $1.5 \mathrm{~kb}$ of CcSp84, were amplified by the polymerase chain reaction (PCR) with primer pairs CcSp84-5F-F/CcSp84-5F-R and CcSp84-3F-F/CcSp84-3F-R (Supplementary Table S1), respectively. The upstream and downstream flanking sequences of CcSp84 were fused with hygromycin $\mathrm{B}$ resistance gene by using the overlapped PCR with the primer pairs CcSp84-5F-F/Hygromycin-R or Hygromycin-F/CcSp84-3F-R (Supplementary Table S1). All the sequences of fragments were confirmed by sequencing analysis. Then the recombinant fragments were co-transformed into protoplasts of WT strain through PEGmediated transformation. The transformed strains were cultured in the TB3 medium with $30 \mu \mathrm{g} / \mathrm{mL}$ hygromycin and then were selected and verified by the PCR with the primer pair External-CcSp84-F/R and Internal-CcPs84-F/R. The Southern blot analysis was performed to confirm the single copy homologous recombination event by using the DIG High Prime DNA Labeling and Detection Starter Kit I, following the manufacturer's protocol (Roche, Germany). The genomic DNA from the WT strain, CcSp31 and CcSp84 deletion mutants, were digested by restriction enzyme $\mathrm{NcoI}$ and then separated by gel electrophoresis. The 
probe fragments for CcSp31 and CcSp84 were acquired by PCR amplification with the primers CcSp31-Pro-F and CcSp31-Pro-R or CcSp84-Pro-F and CcSp84-Pro-R, respectively, and then labeled with DIG.

For complementation, the full length of $C c S p 84$, including the $1.5 \mathrm{~kb}$ native promoter, CcSp84 genomic sequences, and $0.2 \mathrm{~kb}$ terminal sequences and geneticin-resistant cassettes, were co-transformed into the protoplast of the deletion mutant. Then, transformants were selected by $30 \mu \mathrm{g} / \mathrm{mL}$ hygromycin and $50 \mu \mathrm{g} / \mathrm{mL}$ geneticin. The successful complementary strain was named as $\Delta C \operatorname{CSp} 84 / \mathrm{C}$ in this study. A similar strategy was used for the deletion and complementation of CcSp31.

\subsection{Plasmid Construction and Transient Expression}

To determine the eliciting or inhibitory activity of candidate effectors, the coding sequence of $C c S p 84$ without signal peptide (mature type) was amplified from the cDNA library of C. chrysosperma with primer pairs CcSp84-sp-F/CcSp84-R. The plasmid pGR106 was linearized by Cla I/Not I and then ligation with the purified fragment of CcSp84 to obtain the CcSp84-pGR106 recombinant plasmid. The CcSp31-pGR106 recombinant plasmid was constructed in the same way with primer pairs CcSp31-sp-F/CcSp84-R.

In order to verify the influence of different positioning conditions on CcSp84, several mutant types of CcSp84 were generated and cloned into pBINGFP2, including the CcSp84_nls_NES (the NLS sequence was removed, and a nuclear export signal (NES) sequence was added at the C-terminal), CcSp84_nls (the NLS sequence in CcSp84 was removed), and CcSp84_NES (an NES sequence was added at the C-terminal of CcSp84). The original plasmids pGR106 (EV) and pBINGFP2 were kindly provided by Pro. Daolong Dou from Nanjing Agricultural University.

All the recombinant plasmids were transformed into Agrobacterium tumefaciens strain GV3101 by 2500 volts electric shocking [54]. The recombinant strains were cultured in LB liquid medium with $50 \mu \mathrm{g} / \mathrm{mL}$ kanamycin and rifampin resistance at $200 \mathrm{rpm}$ and $2{ }^{\circ} \mathrm{C}$ for $48 \mathrm{~h}$, and then the strain's solution was washed with the $10 \mathrm{mM} \mathrm{MgCl}{ }_{2}$ solution three times, resuspended with MMA buffer $\left(10 \mathrm{mM} \mathrm{MgCl}_{2}, 10 \mathrm{mM}\right.$ 2-ethanesulfonic acid, and $200 \mu \mathrm{M}$ acetosyringone), and cultured for $4 \mathrm{~h}$. The culture solution was then diluted by adjusting the $\mathrm{OD}_{600}$ to 0.4 with MMA buffer and infiltrated into the leaves of $N$. benthamiana with a 1-mL syringe.

\subsection{Confocal Fluorescent Analysis}

After 2 days of agro-infiltration of $A$. tumefaciens GV3101 containing pBINGFP2, CcSp84pBINGFP2, CcSp84_nls-pBINGFP2, CcSp84_NES-pBINGFP2, and CcSp84_nls_NES-pBINGFP2, the leaves of $N$. benthamiana were collected and infiltrated with $5 \mathrm{mg} / \mathrm{mL}$ DAPI (4'6diamidino-2-phenylindole). After $1 \mathrm{~h}$, the leaves were cut into $8 \times 8 \mathrm{~mm}$ pieces and observed by the Laser confocal microscope (Leica SP8, Weztlar, Germany). The GFP fluorescence was excited using $488 \mathrm{~nm}$ laser line.

\subsection{Detection of Reactive Oxygen and Callosum}

To detect the ROS burst, the $N$. benthamiana leaves were stained and shaken in $1 \mathrm{mg} / \mathrm{mL}$ 3, 3'-Diaminobenzidine (DAB) solution (Sigma, USA) for $2 \mathrm{~h}$ after $24 \mathrm{~h}$ post-inoculation. Then, the leaves were discolored with $95 \%$ ethanol and observed by the microscope (Leica DM 2500, Weztlar, Germany). Callose deposition was detected by using aniline blue staining [55]. Agro-infiltrated N. benthamiana leaves were de-stained with $95 \%$ ethanol and then stained with $0.05 \%$ aniline blue in $0.067 \mathrm{~mol} \mathrm{~K}_{2} \mathrm{HPO}_{4}$ buffer overnight and subsequently observed using a Leica DM2500 microscope (Leica, Weztlar, Germany).

\subsection{Pathogenicity Assay}

For the pathogenicity assay, branches of annual susceptible host Populus euramericana were cut to $15 \mathrm{~cm}$ long and sealed by the wax. Then, the branches were scald by a $5 \mathrm{~mm}$ diameter hot soldering iron and inoculated with C. chrysosperma mycelial plugs. After 
inoculation, branches were preserved in $25^{\circ} \mathrm{C}$ and a wet environment. The lesions were photographed and measured at $4 \mathrm{dpi}$. The experiment was repeated three times.

\subsection{RNA Extraction and qRT-PCR Analysis}

The samples of $C$. chrysosperma were collected at $48 \mathrm{~h}$ post culture in PDB, and $N$. benthamiana leaves infiltrated with $A$. tumefaciens GV3101 containing different recombinant plasmids were collected $24 \mathrm{~h}$ post-infiltration. Total RNA was extracted with RNA Easy Fast Plant Tissue Kit (TIANGEN) according to the manufacturer's instructions. Firststrand cDNA was synthesized with 2 ug RNA by ABScript II cDNA First-stand Synthesis Kit (ABclonal). The following qPCR used the qPCR SYBR Green Master Mix (Yeasen). The $\mathrm{Nb}$ Actin was set as endogenous control, the relative expression levels were calculated by the $2-\Delta \Delta \mathrm{Ct}$ method with three independent biological replicates. All primers used in this study were listed in Supplementary Table S1.

Supplementary Materials: The following are available online at https:/ / www.mdpi.com/article/10.3 390/ijms23031614/s1.

Author Contributions: Conceptualization, D.X. and C.T.; methodology, Z.X. and Z.H.; investigation, Z.X. and Z.H.; writing—original draft preparation, Z.X. and Z.H; writing-review and editing, D.X. and C.T.; visualization, Z.X. and Z.H.; supervision, D.X. and C.T.; funding acquisition, D.X. All authors have read and agreed to the published version of the manuscript.

Funding: This work was supported by the National Natural Science Foundation of China (31800540) and the Fundamental Research Funds for the Central Universities (2021ZY15).

Institutional Review Board Statement: Not applicable.

Informed Consent Statement: Not applicable.

Data Availability Statement: Data are contained within the article or Supplementary Material.

Acknowledgments: We thank Daolong Dou from Nanjing Agricultural University for kindly providing the plasmids pGR106-EV, pGR106-Bax, and pBinGFP2.

Conflicts of Interest: The authors declare no conflict of interest.

\section{References}

1. Kepley, J.B.; Reeves, F.B.; Jacobi, W.R.; Adams, G.C. Species associated with cytospora canker on Populus tremuloides. Mycotaxon 2015, 130, 783-805. [CrossRef]

2. Han, Z.; Xiong, D.; Xu, Z.; Liu, T.; Tian, C. The Cytospora chrysosperma Virulence Effector CcCAP1 Mainly Localizes to the Plant Nucleus to Suppress Plant Immune Responses. mSphere 2021, 6, 1. [CrossRef] [PubMed]

3. Tao, D.; Li, P.H.; Carter, J.V.; Ostry, M.E. Relationship of Environmental Stress and Cytospora chrysosperma Infection to Spring Dieback of Poplar Shoots. For. Sci. 1984, 30, 645-651.

4. Biggs, A.R.; Davis, D.D.; Merrill, W. Histopathology of cankers on Populus caused by Cytospora chrysosperma. Can. J. Bot. 1983, 61, 563-574. [CrossRef]

5. Yu, L.; Xiong, D.; Han, Z.; Liang, Y.; Tian, C. The mitogen-activated protein kinase gene CcPmk1 is required for fungal growth, cell wall integrity and pathogenicity in Cytospora chrysosperma. Fungal Genet. Biol. 2019, 128, 1-13. [CrossRef] [PubMed]

6. Guyon, J.C.; Jacobi, W.R.; Mcintyre, G.A. Effects of environmental stress on the development of cytospora canker of aspen. Plant Dis. 1996, 80, 1320-1326. [CrossRef]

7. Fan, X.-L.; Liang, Y.-M.; Ma, R.; Tian, C.-M. Morphological and phylogenetic studies of Cytospora (Valsaceae, Diaporthales) isolates from Chinese scholar tree, with description of a new species. Mycoscience 2014, 55, 252-259. [CrossRef]

8. Wang, Y.; Wang, Y. Oxalic acid metabolism contributes to full virulence and pycnidial development in the poplar canker fungus Cytospora chrysosperma. Phytopathology 2020, 110, 1319-1325. [CrossRef]

9. Xiong, D.; Yu, L.; Shan, H.; Tian, C. CcPmk1 is a regulator of pathogenicity in Cytospora chrysosperma and can be used as a potential target for disease control. Mol. Plant Pathol. 2021, 22, 710-726. [CrossRef]

10. Han, Z.; Yu, R.; Xiong, D.; Tian, C. A Sge1 homolog in Cytospora chrysosperma governs conidiation, virulence and the expression of putative effectors. Gene 2021, 778, 145474. [CrossRef]

11. Jones, J.D.G.; Dangl, J.L. The plant immune system. Nature 2006, 444, 323-329. [CrossRef] [PubMed]

12. Dodds, P.N.; Rathjen, J.P. Plant immunity: Towards an integrated view of plant-pathogen interactions. Nat. Rev. Genet. 2010, 11, 539-548. [CrossRef] [PubMed] 
13. Chisholm, S.T.; Coaker, G.; Day, B.; Staskawicz, B.J. Host-Microbe Interactions: Shaping the Evolution of the Plant Immune Response. Cell 2006, 124, 803-814. [CrossRef] [PubMed]

14. Kunze, G.; Zipfel, C.; Robatzek, S.; Niehaus, K.; Boller, T.; Felix, G. The N Terminus of Bacterial Elongation Factor Tu Elicits Innate Immunity in Arabidopsis Plants. Plant Cell 2004, 16, 3496-3507. [CrossRef] [PubMed]

15. Fliegmann, J.; Mithofer, A.; Wanner, G.; Ebel, J. An ancient enzyme domain hidden in the putative beta-glucan elicitor receptor of soybean may play an active part in the perception of pathogen-associated molecular patterns during broad host resistance. J. Biol. Chem. 2004, 279, 1132-1140. [CrossRef] [PubMed]

16. Wang, Q.; Han, C.; Ferreira, A.O.; Yu, X.; Ye, W.; Tripathy, S.; Kale, S.D.; Gu, B.; Sheng, Y.; Sui, Y.; et al. Transcriptional Programming and Functional Interactions within the Phytophthora sojae RXLR Effector Repertoire. Plant Cell 2011, 23, $2064-2086$. [CrossRef] [PubMed]

17. Białas, A.; Zess, E.K.; De la Concepcion, J.C.; Franceschetti, M.; Pennington, H.G.; Yoshida, K.; Upson, J.L.; Chanclud, E.; Wu, C.-H.; Langner, T.; et al. Lessons in Effector and NLR Biology of Plant-Microbe Systems. Mol. Plant-Microbe Interact. 2018, $31,34-45$. [CrossRef] [PubMed]

18. Chen, J.; Hu, L.; Sun, L.; Lin, B.; Huang, K.; Zhuo, K.; Liao, J. A novel Meloidogyne graminicola effector, MgMO237, interacts with multiple host defence-related proteins to manipulate plant basal immunity and promote parasitism. Mol. Plant Pathol. 2018, 19, 1942-1955. [CrossRef] [PubMed]

19. Dou, D.; Zhou, J.-M. Phytopathogen Effectors Subverting Host Immunity: Different Foes, Similar Battleground. Cell Host Microbe 2012, 12, 484-495. [CrossRef]

20. Yuan, M.; Jiang, Z.; Bi, G.; Nomura, K.; Liu, M.; Wang, Y.; Cai, B.; Zhou, J.-M.; He, S.Y.; Xin, X.-F. Pattern-recognition receptors are required for NLR-mediated plant immunity. Nature 2021, 592, 105-109. [CrossRef] [PubMed]

21. Kang, H.; Nguyen, Q.-M.; Iswanto, A.B.B.; Hong, J.C.; Bhattacharjee, S.; Gassmann, W.; Kim, S.H. Nuclear Localization of HopA1 $1_{\text {Pss61 }}$ Is Required for Effector-Triggered Immunity. Plants 2021, 10, 888. [CrossRef] [PubMed]

22. Zhang, L.; Ni, H.; Du, X.; Wang, S.; Ma, X.; Nürnberger, T.; Guo, H.; Hua, C. The Verticillium-specific protein VdSCP7 localizes to the plant nucleus and modulates immunity to fungal infections. New Phytol. 2017, 215, 368-381. [CrossRef] [PubMed]

23. Boevink, P.C.; Wang, X.; McLellan, H.; He, Q.; Naqvi, S.; Armstrong, M.R.; Zhang, W.; Hein, I.; Gilroy, E.M.; Tian, Z.; et al. A Phytophthora infestans RXLR effector targets plant PP1c isoforms that promote late blight disease. Nat. Commun. 2016, 7, 10311. [CrossRef] [PubMed]

24. Chen, T.; Peng, J.; Yin, X.; Li, M.; Xiang, G.; Wang, Y.; Lei, Y.; Xu, Y. Importin- $\alpha$ s are required for the nuclear localization and function of the Plasmopara viticola effector PvAVH53. Hortic. Res. 2021, 8, 1-12. [CrossRef]

25. Franceschetti, M.; Maqbool, A.; Jiménez-Dalmaroni, M.J.; Pennington, H.G.; Kamoun, S.; Banfield, M.J. Effectors of Filamentous Plant Pathogens: Commonalities amid Diversity. Microbiol. Mol. Biol. Rev. 2017, 81, e00066-16. [CrossRef]

26. Sperschneider, J.; Dodds, P.N.; Gardiner, D.M.; Singh, K.B.; Taylor, J.M. Improved prediction of fungal effector proteins from secretomes with EffectorP 2.0. Mol. Plant Pathol. 2018, 19, 2094-2110. [CrossRef]

27. Wang, D.; Tian, L.; Zhang, D.; Song, J.; Song, S.; Yin, C.; Zhou, L.; Liu, Y.; Wang, B.; Kong, Z.; et al. Functional analyses of small secreted cysteine-rich proteins identified candidate effectors in Verticillium dahliae. Mol. Plant Pathol. 2020, 21, 667-685. [CrossRef]

28. Chapman, S.; Kavanagh, T.; Baulcombe, D. Potato virus $X$ as a vector for gene expression in plants. Plant J. $1992,2,549-557$.

29. Broekaert, W.F.; Delauré, S.L.; De Bolle, M.F.; Cammue, B.P. The Role of Ethylene in Host-Pathogen Interactions. Annu. Rev. Phytopathol. 2006, 44, 393-416. [CrossRef]

30. Gupta, A.; Bhardwaj, M.; Tran, L.-S. Jasmonic Acid at the Crossroads of Plant Immunity and Pseudomonas syringae Virulence. Int J. Mol. Sci. 2020, 21, 7482. [CrossRef]

31. Situ, J.; Jiang, L.; Fan, X.; Yang, W.; Li, W.; Xi, P.; Deng, Y.; Kong, G.; Jiang, Z. An RXLR effector PlAvh142 from Peronophythora litchii triggers plant cell death and contributes to virulence. Mol. Plant Pathol. 2020, 21, 415-428. [CrossRef] [PubMed]

32. Pieterse, C.M.J.; Van der Does, D.; Zamioudis, C.; Leon-Reyes, A.; Van Wees, S.C.M. Hormonal Modulation of Plant Immunity. Annu. Rev. Cell Dev. Biol. 2012, 28, 489-521. [CrossRef] [PubMed]

33. Park, C.J.; Ronald, P.C. Cleavage and nuclear localization of the rice XA21 immune receptor. Nat. Commun. 2012, 3, 920. [CrossRef] [PubMed]

34. Deslandes, L.; Rivas, S. The plant cell nucleus: A true arena for the fight between plants and pathogens. Plant Signal. Behav. 2011, 6, 42-48. [CrossRef]

35. Jeon, H.; Kim, W.; Kim, B.; Lee, S.; Jayaraman, J.; Jung, G.; Choi, S.; Sohn, K.H.; Segonzac, C. Ralstonia solanacearum Type III Effectors with Predicted Nuclear Localization Signal Localize to Various Cell Compartments and Modulate Immune Responses in Nicotiana spp. Plant Pathol. J. 2020, 36, 43-53. [CrossRef]

36. Cui, H.; Tsuda, K.; Parker, J.E. Effector-Triggered Immunity: From Pathogen Perception to Robust Defense. Annu. Rev. Plant Biol. 2015, 66, 487-511. [CrossRef]

37. Gui, Y.-J.; Chen, J.-Y.; Zhang, D.-D.; Li, N.-Y.; Li, T.-G.; Zhang, W.-Q.; Wang, X.-Y.; Short, D.P.G.; Li, L.; Guo, W.; et al. Verticillium dahliae manipulates plant immunity by glycoside hydrolase 12 proteins in conjunction with carbohydrate-binding module 1. Environ. Microbiol. 2017, 19, 1914-1932. [CrossRef] 
38. Qi, T.; Guo, J.; Liu, P.; He, F.; Wan, C.; Islam, A.; Tyler, B.M.; Kang, Z.; Guo, J. Stripe Rust Effector PstGSRE1 Disrupts Nuclear Localization of ROS-Promoting Transcription Factor TaLOL2 to Defeat ROS-Induced Defense in Wheat. Mol. Plant 2019, 12, 1624-1638. [CrossRef]

39. Ma, Z.; Song, T.; Zhu, L.; Ye, W.; Wang, Y.; Shao, Y.; Dong, S.; Zhang, Z.; Dou, D.; Zheng, X.; et al. A Phytophthora sojae Glycoside Hydrolase 12 Protein Is a Major Virulence Factor during Soybean Infection and Is Recognized as a PAMP. Plant Cell 2015, 27, 2057-2072. [CrossRef]

40. Zhu, W.; Ronen, M.; Gur, Y.; Minz-Dub, A.; Masrati, G.; Ben-Tal, N.; Savidor, A.; Sharon, I.; Eizner, E.; Valerius, O.; et al. BcXYG1, a Secreted Xyloglucanase from Botrytis cinerea, Triggers Both Cell Death and Plant Immune Responses. Plant Physiol. 2017, 175, 438-456. [CrossRef]

41. Wu, C.-H.; Derevnina, L.; Kamoun, S. Receptor networks underpin plant immunity. Science 2018, 360, 1300-1301. [CrossRef] [PubMed]

42. Doehlemann, G.; Ökmen, B.; Zhu, W.; Sharon, A. Plant pathogenic fungi. Microbiol. Spectr. 2017, 5, 14. [CrossRef] [PubMed]

43. Kanja, C.; Hammond-Kosack, K.E. Proteinaceous effector discovery and characterization in filamentous plant pathogens. Mol. Plant Pathol. 2020, 21, 1353-1376. [CrossRef] [PubMed]

44. Xu, Q.; Tang, C.; Wang, X.; Sun, S.; Zhao, J.; Kang, Z.; Wang, X. An effector protein of the wheat stripe rust fungus targets chloroplasts and suppresses chloroplast function. Nat. Commun. 2019, 10, 5571. [CrossRef] [PubMed]

45. Tian, H.; MacKenzie, C.I.; Rodriguez-Moreno, L.; Berg, G.C.M.V.D.; Chen, H.; Rudd, J.J.; Mesters, J.R.; Thomma, B.P.H.J. Three LysM effectors of Zymoseptoria tritici collectively disarm chitin-triggered plant immunity. Mol. Plant Pathol. 2021, $22,683-693$. [CrossRef] [PubMed]

46. Yi, S.Y.; Shirasu, K.; Moon, J.S.; Lee, S.-G.; Kwon, S.-Y. The Activated SA and JA Signaling Pathways Have an Influence on flg22-Triggered Oxidative Burst and Callose Deposition. PLoS ONE 2014, 9, e88951. [CrossRef] [PubMed]

47. An, C.; Mou, Z. Salicylic Acid and its Function in Plant Immunity. J. Integr. Plant Biol. 2011, 53, 412-428. [CrossRef]

48. Betsuyaku, S.; Katou, S.; Takebayashi, Y.; Sakakibara, H.; Nomura, N.; Fukuda, H. Salicylic acid and jasmonic acid pathways are activated in spatially different domains around the infection site during effector-triggered immunity in Arabidopsis thaliana. Plant Cell Physiol. 2018, 59, 439. [CrossRef] [PubMed]

49. Gimenez-Ibanez, S.; Boter, M.; Fernández-Barbero, G.; Chini, A.; Rathjen, J.; Solano, R. The Bacterial Effector HopX1 Targets JAZ Transcriptional Repressors to Activate Jasmonate Signaling and Promote Infection in Arabidopsis. PLoS Biol. 2014, 12, e1001792. [CrossRef]

50. Zhou, Z.; Wu, Y.; Yang, Y.; Du, M.; Zhang, X.; Guo, Y.; Li, C.; Zhou, J.-M. An Arabidopsis Plasma Membrane Proton ATPase Modulates JA Signaling and Is Exploited by the Pseudomonas syringae Effector Protein AvrB for Stomatal Invasion. Plant Cell 2015, 27, 2032-2041. [CrossRef]

51. Thordal-Christensen, H.; Birch, P.R.J.; Spanu, P.D.; Panstruga, R. Why did filamentous plant pathogens evolve the potential to secrete hundreds of effectors to enable disease? Mol. Plant Pathol. 2018, 19, 781-785. [CrossRef] [PubMed]

52. Catlett, N.L.; Lee, B.N.; Yoder, O.C.; Turgeon, B.G. Split-marker recombination for efficient targeted deletion of fungal genes. Fungal Genet. Rep. 2003, 50, 9-11. [CrossRef]

53. Goswami, R.S. Targeted Gene Replacement in Fungi Using a Split-Marker Approach. Programmed Necrosis 2012, 835, $255-269$.

54. Li, W.; Cao, J.; Xu, Y.; Cai, X. Artificial Agrobacterium tumefaciens strains exhibit diverse mechanisms to repress Xanthomonas oryzae pv. oryzae-induced hypersensitive response and nonhost resistance in Nicotiana benthamiana. Mol. Plant Pathol. 2016, 18, 489. [CrossRef] [PubMed]

55. Schenk, S.T.; Hernández-Reyes, C.; Samans, B.; Stein, E.; Neumann, C.; Schikora, M.; Reichelt, M.; Mithöfer, A.; Becker, A.; Kogel, K.-H.; et al. N-Acyl-Homoserine Lactone Primes Plants for Cell Wall Reinforcement and Induces Resistance to Bacterial Pathogens via the Salicylic Acid/Oxylipin Pathway. Plant Cell 2014, 26, 2708-2723. [CrossRef] [PubMed] 